\title{
Possibilités écotoxicologiques des précipitations acides pour le saumon dans 4 rivières à la Côte Nord du fleuve Saint-Laurent
}

\author{
R. Van Coillie ${ }^{1}$ \\ D. Brouard ${ }^{2}$ \\ M. Lachance 2 \\ Y. Vigneault ${ }^{3}$
}

Mots-clés : écotoxicologie, précipitations acides, Salmo salar.

Les précipitations acides régionales ( $\mathrm{pH}$ moyens des pluies et neiges au sol $; 4,1$ et 4,6 ) entraînent les phénomènes suivants dans les eaux oligotrophes des 4 rivières étudiées : (1) déficit d'alcalinité, laquelle déjà faible devient insuffisante pour neutraliser les apports d'acidité ; (2) amplification des concentrations d'aluminium suite à un lessivage acide des alumino-silicates du bouclier canadien ; (3) augmentation parallèle des teneurs en fer, manganèse et cuivre. Ces 3 phénomènes sont surtout prononcés à la fonte printanière des neiges. A cette période, le pH descend quelque peu sous 6,0 aux têtes du bassin, la concentration d'aluminium s'accroît d'amont à aval jusqu'à atteindre $355 \mu \mathrm{g} \mathrm{Al/L}$ en moyenne (maximum : $894 \mu \mathrm{g} / \mathrm{L}$ ) et, similairement, celles du fer, manganèse et cuivre s'élèvent jusqu'à $317 \mu \mathrm{g} \mathrm{Fe} / \mathrm{L}, 16 \mu \mathrm{g} M \mathrm{Mn} / \mathrm{L}$ et $11 \mu \mathrm{g}$ $\mathrm{Cu} / \mathrm{L}$ en moyenne (maxima respectifs : $376 \mu \mathrm{g} / \mathrm{L}, 31 \mu \mathrm{g} / \mathrm{L}, 27 \mu \mathrm{g} / \mathrm{L}$ ). A ce moment, le saumon local est au début du stade alevin, lequel s'avère particuljèrement sensible. Les risques écotoxicologiques des 3 phénomènes précités sont dès lors examinés pour Salmo salar au commencernent de son développement. Actuejlement, l'aluminium et le cuivre représentent un danger écotoxicologique pour le saumon dans ces rivières : si les précipitations acides persistent, un pH plus bas (5.5 à 6,0) et le fer deviendront deux aut res facteurs dangereux qui s'ajouteront. Bref, le développement de Salmo salar risque d'être progressivement menacé dans ces rivières en cours d'acidification.

Ecotoxicological possibilities of acid precipitations for salmon in 4 rivers draining the north coast of the St. Lawrence river

Keywords : ecotoxicology, acid precipitations, Salmo salar.

Regional acid precipitations (average $\mathrm{pH}$ of rain and snow on the ground $: 4,1$ and 4,6 ) produce the following phenomena in the oligotrophic water of 4 rivers studied : (1) an alkalinity deficit, which already low becomes insufficient to neutralize added acidity, (2) higher concentrations of aluminium following an acid leaching of the alumino-silicates in the canadian shield ; (3) a similar increase in levels of iron, manganese and copper. These 3 phenomena are most evident during the spring snow melt. At this time $\mathrm{pH}$ levels fall slightly below 6,0 at the heads of the basin, the concentration of aluminum inc reases from upstream to downst ream at taining an average of $355 \mu \mathrm{g} \mathrm{Al} / \mathrm{L}$ (maximum : $894 \mu \mathrm{g} / \mathrm{L}$ ) and, simi. larly, levels of iron, manganese and copper rise to $317 \mu \mathrm{g} \mathrm{Fe} / \mathrm{L}, 16 \mu \mathrm{g} \mathrm{Mn} / \mathrm{L}$ and $11 \mu \mathrm{g} \mathrm{Cu} / \mathrm{L}$ on the average (respective maxima ; $376 \mu \mathrm{g} / \mathrm{L}, 31 \mu \mathrm{g} / \mathrm{L}, 27 \mu \mathrm{g} / \mathrm{L}$ ). At this time salmon are in the early alevin stages of their development, a very sensitive time in the life of the fish. The ecotoxicological risks of the 3 afore-mentioned phenomena are therefore examined for Salmo salar in the early stages of its development. At present, both aluminium and copper present an ecotoxicological danger for salmon in these rivers : if acid precipitations continue, a lower $\mathrm{pH}(5,5$ to 6,0$)$ and iron will also become hazardous. Briefly, the development of Saimo salar in these rivers becomes increasingly threatened through progressive acidification of the water.

1. Conseil de la Science et de la Technologie, conseil exécutif du gouvernement du Québec, 1275 Boulevard Charest, Québec. Ginacd, Canada.

2. Institut National de la Recherche Scientifique, Université du Québec, 2700 rue Einstein, Sainte-Foy, Québec, G1V 4C7, Canada.

3. Péches et Océans Canada. Direction de la recherche, 901 Cap Diamant, C.P. 1550, Québec. Canada.

\section{1. - Introduction}

Les rivières de la Côte Nord du fleuve SaintLaurent en aval de la Malbaie restent réputées pour le saumon. Parmi celles-ci, Salmo salar continue à utiliser les rivières Petit Saguenay et SainteMarguerite et, du moins en deçà de ses chutes 
situées à $3 \mathrm{~km}$ en amont du lac aux Brochets, la rivière des Petits Escoumins (Carter 1968). Il se retrouve aussi dans la rivière des Escoumins où, malgré une exploitation forestière du bassin versant et un flottage de bois depuis 1937, l'aménagement de deux passes migratoires à l'embouchure en $1965-1966$ et l'ensemencement d'au moins 150000 alevins et 23000 saumoneaux depuis 1961 par le ministère du Loisir, de la Chasse et de la Pêche du Québec favorisent une ré-implantation de l'espèce (Hovington \& al. 1978).

Dans ces rivières, la survie de Salmo salar est actuellement protégée voire favorisée par des actions d'aménagements et de contrôles relativement efficaces. Toutefois, le saumon qu'on y trouve subit les effets induits par les précipitations acides qui se déversent dans la région (Altshuller \& Mc Bean 1980).

Parmi ces effets, nous examinerons ceux qui peuvent influencer les étapes du cycle vital du saumon en eau $x$ douces et nous évaluerons le risque qu'il soit affecté à long terme par les précipitations acides dans les quatre rivières.

\section{2. - Effets physico-chimiques des précipitations acides dans les quatre rivières}

Afin de préciser ces effets, un échantillonnage a été fait durant l'hiver, le printemps et l'automne 1981 à 5 stations dont la localisation est présentée sur la figure 1 .

Le choix des stations fut dicté selon leur potentiel comme site propice à la reproduction du saumon. La méthode d'évaluation de ces sites fut basée principalement sur une caractérisation par photointerprétation des tronçons de rivières pour leur morphologie du cours, leurs faciès d'écoulement et la granulométrie des matériaux constituant leur lit. Ceci a conduit à la classification des tronçons et au choix de ceux représentant le meilleur potentiel de frai. Pour chacune des rivières, une seule station fut retenue dans ces cours d'eau ; la rivière des Escoumins fait cependant exception puisqu'une seconde station, située à proximité de son embouchure, fut ajoutée afin d'estimer la variation amont-aval de la composition physico-chimique de ses eaux. A ces stations, les superficies des bassins sont les suivantes :
PS $_{1}$ : $286 \mathrm{~km}^{2}$ du bassin du Petit Saguenay, soit $35 \%$

$\mathrm{SM}_{1}: 818 \mathrm{~km}^{2}$ du bassin de la Sainte-Marguerite, soit $81 \%$

ES $_{1}$ : $359 \mathrm{~km}^{2} \mathrm{du}$ bassin des Escoumins, soit $45 \%$

$\mathrm{ES}_{2}: 789 \mathrm{~km}^{2} \mathrm{du}$ bassin des Escoumins, soit $99 \%$

$\mathrm{PE}_{1}: 108 \mathrm{~km}^{2}$ du bassin des Petits Escoumins, soit $78 \%$.

Les modalités de l'échantillonnage, les mesures et méthodes analytiques adoptées (APHA \& al. 1980) ainsi que les résultats bruts acquis sont détaillés dans 2 ouvrages antérieurs (Brouard \& al 1981 et 1982. Ces résultats ont été regroupés par saison et par station ; hormis ceux de la neige et de la pluie, ils ont été pondérés en fonction du débit selon l'équation suivante :

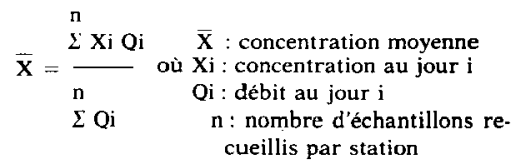

Le Tableau I présente les moyennes obtenues par paramètre, saison et station pour les eaux de surface des 4 rivières. Pour certains paramètres, il y a des variations amont-aval très nettes : celles-ci sont illustrées au tableau II.

Tableau II. - Variations amont-aval pour certains parametres physico-chimiques à la rivière des Escoumins durant les trois saisons échantillonnées (1981)

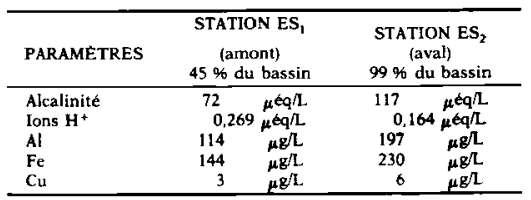

L'étude physico-chimique a notamment permis d'expliciter les points suivants (Van Coillie \& al 1982 a).

1) L'acidité des p'uies ( $\mathrm{pH}: 3,8$ à 4,6 avec moyenne à 4,1 ) et des neiges ( $\mathrm{pH}: 4,4$ à 4,9 avec moyenne à 4,6 au sol) occasionne un apport excessif d'ions $\mathrm{H}^{+}$ dans les 4 rivières mais l'alcalinité de celles-ci, bien qu'elle soit basse $\left(\leqslant 200_{\mu}\right.$ éq/L, indication normative 


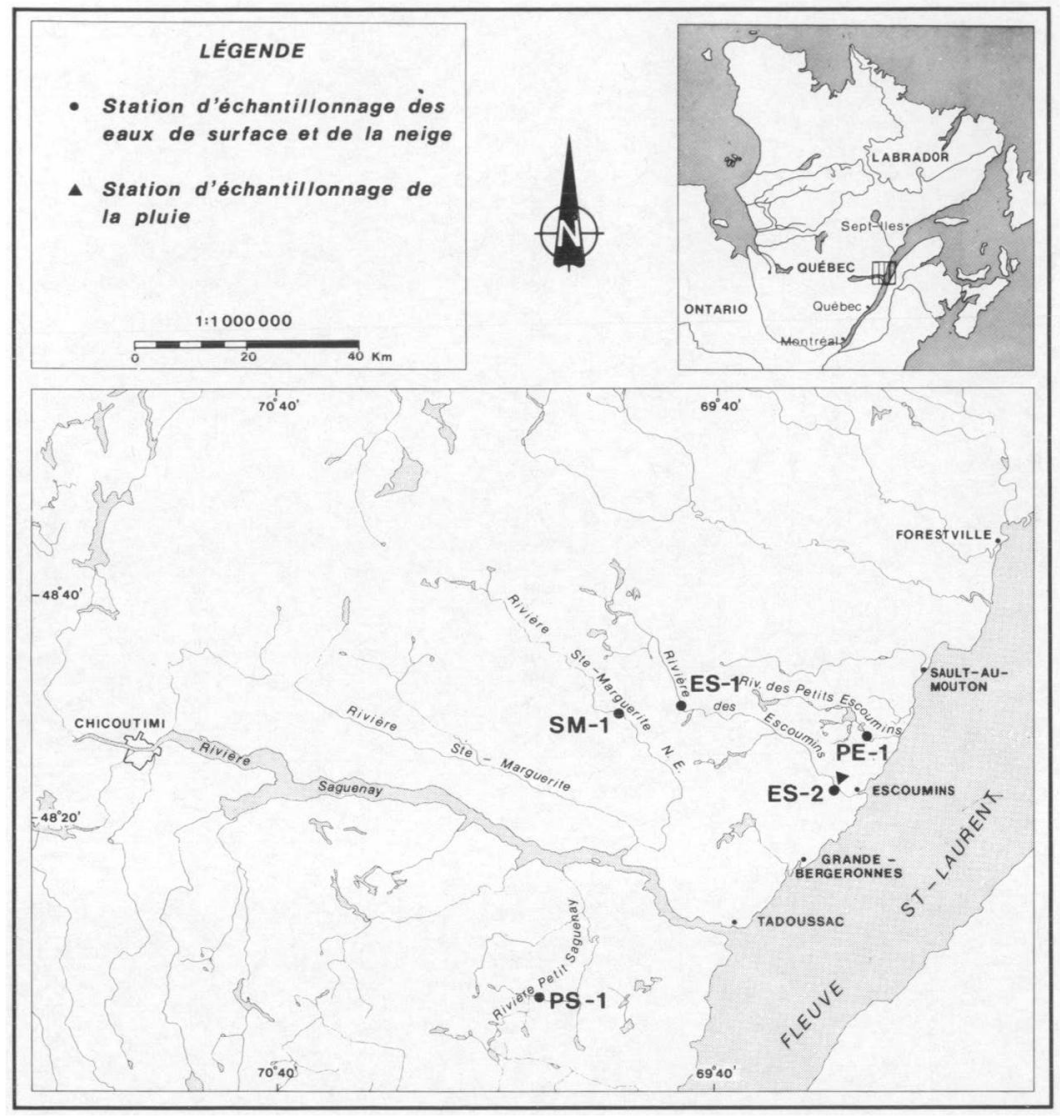

Fig. 1. Situation des rivières et des stations étudiées. 
Tableau 1. - Valeurs moyennes pondérées par le débit pour les paramètres physico-chimiques des eaux de quatre rivières à saumons à la Cóte Nord du Saint-Laurent (1981)

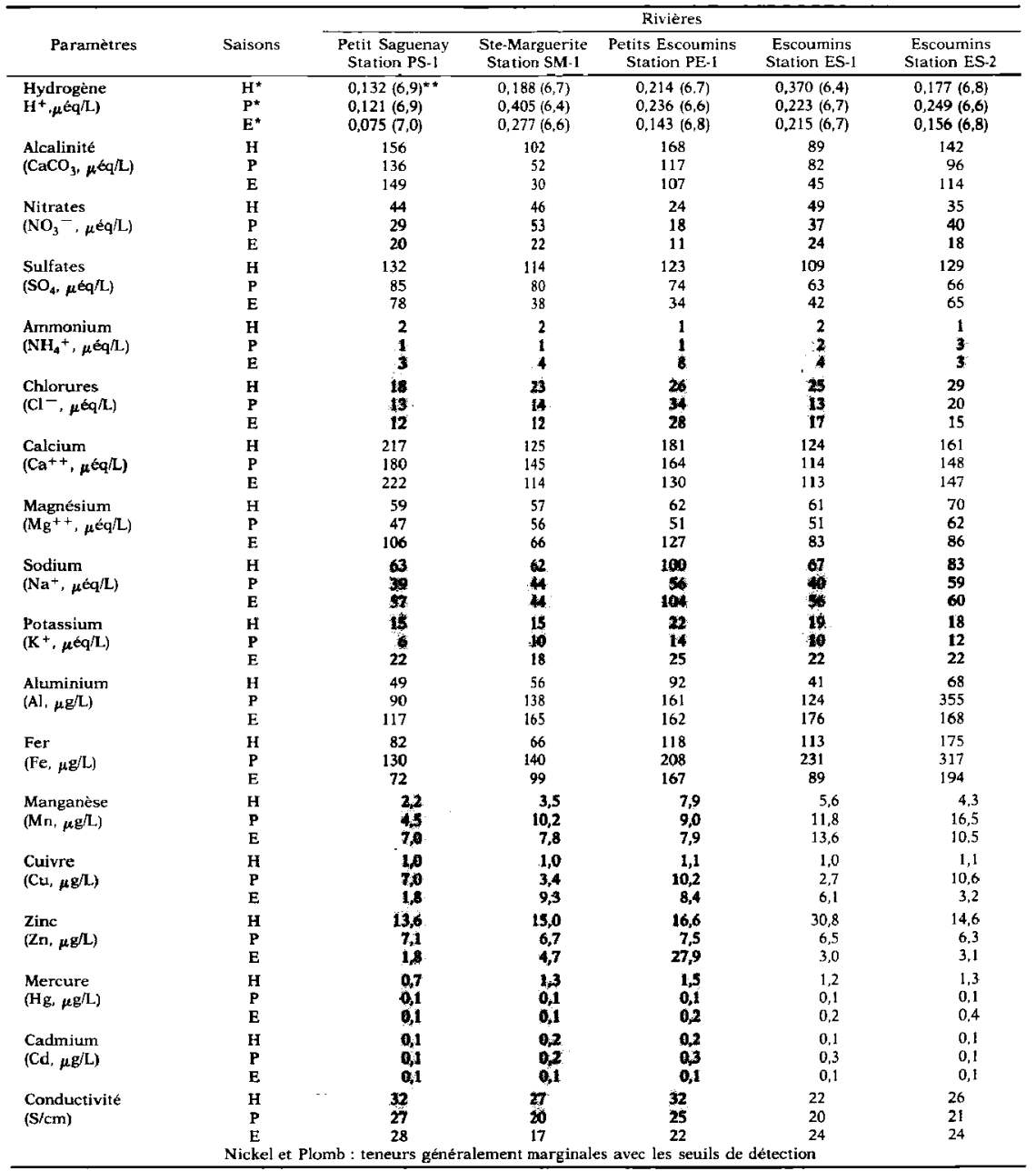

* Nombre d'échantillons: Hiver $(\mathrm{H}): \mid \mathrm{N}=3$

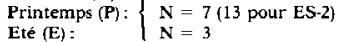

** Valeurs de pH

$* * *$ Echantillons filtrés $(\leqslant 0,45 \mu \mathrm{m})$ 
citée par Altshuller et Mc Bean), neutralise en grande partie cet apport de telle sorte qu'on n'y décèle pas une chute importante des $\mathrm{pH}$ (6,4 à 7,0 avec moyenne à 6,7). Toutefois, en montant vers la tête des bassins, on s'aperçoit que l'alcalinité devient encore plus faible (tableau II), ce qui rend les eaux plus sensibles aux précipitations acides. Par exemple, à $60 \mathrm{~km}$ de son embouchure (site nettement en amont de $\mathrm{ES}_{1}$ ), la rivière des Escoumins a une alçalinité réduite à $32 \mu$ éq/L et un $\mathrm{pH}$ abaissé à 5,9 (Brouard \& Lachance, 1982). En fait, malgré que leur $\mathrm{pH}$ se révèle peu acide, les 4 rivières font partie de celles qu'on évalue comme étant en cours d'acidification si l'on considère, d'une part, leur teneur élevée en sulfates $(\geqslant 60 \mu$ éq $/ L$, valeur en deçà de laquelle se situent généralement les concentrations de sulfates dans les eaux du bouclier canadien non affectées par les précipitations acides selon Harvey $\&$ al 1981) et, d'autre part, leur déficit en alcalinite qui peut être évalué par la teneur en $\mathrm{Ca}+++\mathrm{Mg}^{++}$ $-\mathrm{HCO}_{3}++$ - (Dillon \& al 1980, Harvey \& al 1981).

2) Les concentrations en aluminium apparaissent très hautes dans les eaux étudiées par rapport à celles qui prévalent normalement dans les eaux naturelles (20 à $100 \mu \mathrm{g} \mathrm{Al} / \mathrm{L}$, d'après Schofield \& Trojnar, 1980). Notons ici que ce métal de même que les autres métaux traces se retrouvent dans les eaux non seulement sous forme ionique mais aussi et parfois principalement sous forme particulaire et/ou associée avec des substances organiques (Alabaster \& Lloyd 1980, Driscoll \& al. 1980) : il convient dès lors d'exprimer leurs teneurs en $\mu \mathrm{g} / \mathrm{L}$ plutôt qu'en $\mu$ éq/L. Le surcroît constaté pour l'aluminium est vraisemblablement relié à des échanges entre des ions $\mathrm{H}^{+}$en excès dans les eaux acides de ruisselle. ment et des alumino-silicates du bouclier canadien granitique (Harvey \& al 1981).

3) A moindre degré, il y a aussi un surcroît analo. gue dans plusieurs cas pour Fe, Mn et Cu dans les 4 rivières si l'on tient compte des concentrations normales pour ces métaux en eaux naturelles oligotrophes $(200 \mu \mathrm{g} \mathrm{Fe} / \mathrm{L}, 3 \mu \mathrm{g} \mathrm{Mn} / \mathrm{L}$ et $5 \mu \mathrm{g} \mathrm{Cu} / \mathrm{L}$ selon Alabaster \& Lloyd 1980; Harvey \& al 1981).

Ces effets physico-chimiques des précipitations acides risquent-ils de faire disparaitre à long terme le saumon des 4 rivières? Pour répondre à cette question, trois approches complémentaires peuvent être envisagées. En premier lieu, à partir des nombreuses informations disponibles à ce sujet dans la littérature scient ifique pour les salmonidés de Scandinavie et d'autres régions affectées par les préci- pitations acides, on considère s'il y a danger à long terme pour la survie régionale de Salmo salar. En second lieu, des bioessais aident à préciser les réponses de cette espèce à divers facteurs locaux interreliés tels que $\mathrm{pH}$, alcalinité, aluminium et matière organique. Enfin, la vérification de ces réponses in sizu et des études approfondies des saumons dans ces rivières permettent d'intégrer les deux autres approches. La suite de l'exposé traitera surtout de la première des 3 approches prédécrites, la seconde faisant l'objet d'études en cours (Thellen \& Van Coillie 1982) et la troisième étant encore à l'état de projet.

\section{3. - Facteurs alcalinité et pH}

L'influence de ces 2 facteurs interreliés va être précisée aux différentes étapes successives du développement initial du saumon (parfois, on l'examinera aussi, à titre de comparaison, pour d'autres salmonidés), car c'est surtout lors de ce développement initial que le saumon s'avère sensible aux effets des précipitations acides (SNSF 1980).

Le frai du saumon s'effectue vraisemblablement au cours de la seconde quinzaine d'octobre dans les 4 rivières concernées. De fait, des biologistes du gou. vernement du Québec (MLCP) ont recueilli, le 20 octobre 1981, des milliers d'œufs provenant de saumons dans une rivière de la région, à savoir la rivière du Gouffre. A la même période, à la rivière Mistas. sini près de Baie Comeau, des cufs de saumon ont été observés dans le gravier de plusieurs sites de frai que des géniteurs occupaient encore. Or, les rivières en cause dans le présent travail sont situées presque à mi-chemin, entre ces deux cour's d'eau. Bien que l'automne n'ait pu être inclus dans les saisons couvertes par l'étude locale des effets physico. chimiques des pluies acides, la possibilité que cellesci aient alors une influence sur le frai du saumon dans ces rivières doit être envisagée. Cette influence surviendrait surtout dans les parties supérieures des bassins où l'alcalinité devient trop faible à leur tête pour neutraliser complètement un apport excessif d'ions $\mathrm{H}^{+}$, tel que mentionné plus haut. Un $\mathrm{pH}$ voisin de 5,5 n'y est dès lors pas impossible. A ce $\mathrm{pH}$, Menendez (1976) a constaté que la production et la fertilisation des œufs sont nettement réduites pour le salmonidé Salvelinus fontinalis. Ceci peut 
être associé à plusieurs phénomènes complémentaires résumés ci-après.

- La protéosynthèse du vitellus devient plus faible en milieu acide chez les poissons ; par exemple, lorsque le $\mathrm{pH}$ ambiant se situe à 5,5 au lieu de 6,7 durant 20 jours, il y a une diminution de la vitellogenèse protéique qui entraîne une baisse de $85 \%$ dans la formation des ceufs matures à partir des oogonies chez Jordanella floridae (Ruby \& al 1977).

- Les femelles ont également une insuffisance en calcium dans leur sérum pour la maturation des oocytes quand elle vivent en eaux acides, laquelle insuffisance est davantage reliée à l'acidité qu'à la teneur en $\mathrm{Ca}^{++}$dans celles-ci; signalons ici que le rapport sérique $\mathrm{Ca}$ femelle/ $\mathrm{Ca}$ mâle des poissons descend de 2,0 en conditions normales ( $\mathrm{pH} \mathrm{6,7)} \mathrm{à} \mathrm{1,4}$ en conditions modérément acides (pH 5,3) (Lockhart \& Lutz 1977, Fromm 1980).

- Le dépôt des œufs peut aussi être considérablement inhibé (plus de $50 \%$ ) à des $\mathrm{pH}$ 5,5-6,0 chez les poissons; par la suite, une pénétration excessive d'ions $\mathrm{H}^{+}$dans le liquide périvitellin entre l'ooplasme et la membrane des ceufs affecte la survie et la fertilité de ceux-ci (Lee \& Gerkind 1980, Harvey \& al 1981).

Etant donné que, de manière générale, Salmo salar et Salmo gairdneri s'avèrent beaucoup moins tolérants aux $\mathrm{pH}$ acides que Salvelinus fontinalis et Salmo trutta parmi les salmonidés (Johansson \& al 1977 : Grande \& al 1978) et que la plupart des espèces dans les autres familles (Harvey \& al 1981), le saumon est sensible à des $\mathrm{pH}$ quelque peu plus élevés que ceux affectant la majorité des autres espèces. Dans ces conditions, compte tenu de ce qui précède, le frai du saumon commencerait à être inhibé à $\mathrm{pH} \leqslant 6,0$.

Après le frai, l'incubation des ceufs de saumon durerait environ quatre mois; Battle (1944) mentionne 140 jours pour une température ambiante de $2^{\circ} \mathrm{C}$ et Carrick (1979) fait état d'une période moyenne de 115 jours dans une eau à $4^{\circ} \mathrm{C}$. A la station de pisciculture de Tadoussac, qui se trouve à proximité des sites étudiés, les auges d'incubation sont alimentées par les eaux d'un ruisseau local et l'éclosion des œufs de saumon fertilisés à la seconde quinzaine d'octobre s'effectue au début mars. Cette donnée permet de penser que l'incubation des cufs de Salmo salar pourrait bien se terminer à la même époque dans les 4 rivières concernées. Considérons à présent la possibilité qu'un $\mathrm{pH}$ modérément acide
$(\mathrm{pH} \leqslant 6,0)$ soit présent durant l'incubation des œufs de salmonidés à la tête de ces rivières. Les $\mathrm{pH}$ audessous desquels le développement et l'éclosion de ces cufs sont affectés, à une température de 4 à $5^{\circ} \mathrm{C}$, varient selon les espèces. Ainsi, pour Salmo salar et Salmo gairdneri, on rapporte des $\mathrm{pH}$ de 5,0 à 5,5 (Bua \& Snekvik 1972, Kwain, 1975, Johansson \& al 1977) alors que, pour Salvelinus fontinalis et Salmo trutta, on fait mention d'un pH plus acide entre 4,5 et 5,0 (Bua \& Snekvik, 1972, Menendez 1976). D'autres auteurs prétendent toutefois que les œufs de ces différentes espèces ne sont affectés qu'à des pH inférieurs à 4,5 (Carrick 1979; Grande \& al. 1978). La sensibilité des œufs de saumon aux pH acides s'observe, selon Daye (1981), surtout pendant la période de la pré-organogenèse. Cette période qui se déroule avant le stade où les yeux de l'embryon deviennent perceptibles durerait environ 70 jours pour les saumons de la Cóte Nord, si on se fie aux données provenant de la station de pisciculture de Tadoussac. Lors de cette pré-organogenèse, des eaux acides entraînent une acidose du liquide périvitellin, ce qui induit une corrosion de l'ectoderme embryonnaire (Daye \& Garside 1980).

L'éclosion des œufs se révèle également une étape critique. De fait, l'enzyme d'éclosion, dite " chorionase", sécrétée par l'embryon dans le liquide périvitellin et destinée à digérer la capsule de l'œuf, a une activité réduite à $10 \%$ chez le saumon, lorsque le $\mathrm{pH}$ du milieu et, subséquemment, celui du liquide périvitellin descendent à 5,2 ; ceci provient du fait que le $\mathrm{pH}$ d'activité optimale pour cette enzyme est équivalent à 8,0 (Peterson \& al. 1980). Parallèlement, suite à des coagulations partielles, en milieu acide, la capsule chorionaire devient plus opaque et plus difficile à hydrolyser. En pareil cas, de nombreux embryons ne parviennent à libérer que leur région caudale lors de l'éclosion tandis que leur tête et leur sac vitellin restent emprisonnés dans le chorion. A un pH de 5,0-5,5, le retard de l'éclosion qui s'ensuit est de 7 jours avec une mortalité de $30 \%$ (Peterson \& al. 1980). Les diverses données précédentes soutiennent que si le $\mathrm{pH}$ descend à 5,5 aux têtes des qua. tre rivières concernées, le développement et l'éclo. sion des œufs de saumon peuvent y être partiellement affectés. Une telle situation est particulièrement à considérer pour l'éclosion locale durant la première quinzaine de mars. En effet, le début du lessivage du manteau nival à cette période libère, avant les crues printanières, un surcroît d'ions $\mathbf{H}^{+}$ 
et $\mathrm{SO}_{4}^{-}-$dans les eaux de surface de la région (Lachance \& al 1982).

Dès la fin de l'éclosion, les alevins de saumon s'adaptent progressivement à une vie autonome tout en épuisant les réserves de leur sac vitellin. Pendant cette adaptation, la fonte des neiges et les pluies printanières peuvent occasionner, surtout à la tête des 4 rivières, une acidification modérée du milieu aquatique comme cela a été constaté ailleurs dans des eaux non tamponnées par une alcalinité élevée due au calcaire (Jones \& Bisson 1980. SNSF 1980, Harvey \& al 1981). Les alevins de salmonidés présents à l'amont des 4 rivières seraient alors affectés si les $\mathrm{pH}$ deviennent inférieurs à pH 5,0 - 5,5 selon des études faites en laboratoire et en pisciculture (Alabaster \& Lloyd, 1980 ; Farmer \& al, 1980, SNSF 1980, Nelson, 1982). L'étape la plus sensible correspond à la période où les alevins de saumon étant devenus avésiculés, par résorbtion de leur sac vitellin. développent une nutrition exogène, spécialement lors des troisième et quatrième semaines de cette nutrition durant lesquelles un $\mathrm{pH}$ voisin de 5,0 peut induire $38 \%$ de mortalité (Farmer \& al. 1980). Un pH proche de 5,0 ralentit aussi la croissance des alevins chez Salmo salar; après quatre semaines, par exemple, celle-ci est réduite de $60 \%$ en poids et de $20 \%$ en longueur (Farmer \& al, 1980). Des observations analogues ont été faites à $\mathrm{pH} 4,5-5,0$ pour Salmo trutta (Leivestad \& al. 1976) et pour Salvelinus fontinalis (Muniz \& Leivestad 1979). Cependant, chez cette dernière espèce, il y a une variation de la sensibilité aux $\mathrm{pH}$ acides selon les souches étudiées. Ainsi, Menendez (1976) a constaté que la croissance et la survie des alevins d'omble de fontaine qu'il utilisait pour ses expériences étaient réduites à des $\mathrm{pH}$ de 5,5-6,5 tandis que des souches d'omble étudiées par Robinson \& al (1976) résistaient à des pH de 4,5-5,5. En plus d'une croissance diminuée, un $\mathrm{pH}$ voisin de 5,0 induit également divers autres changements chez les alevins de salmonidés, entre autres une inhibition de la pigmentation (insuffisance de mélanine), une réduction de la calcification des structures ostéoïdes malgré une teneur normale de $\mathrm{Ca}^{++}$dans le sérum et, enfin, un abaissement du rythme cardiaque (Daye 1981, Nelson 1982).

Le mécanisme par lequel les salmonidés sont affectés au stade alevin et aux stades ultérieurs par des excès d'ions $\mathrm{H}^{+}$perturbe plusieurs niveaux physiologiques. Il y a d'abord les branchies et, à moindre degré, la peau qui sont modifiées par des pH 5,0 à 5,5: leurs cellules épithéliales d'échange se détachent ou se nécrosent, leurs cellules à mucus sont en hypertrophie avec surproduction de mucus et des engorgements et/ou coagulations de mucus se développent en surface (Daye \& Garside 1980). Simultanément, les processus d'osmorégulation à ces lieux d'échange sont désorganisés : suite à leur grande perméabilité aux ions $\mathrm{H}^{+}$, un influx anormalemment élevé de ceux-ci survient au détriment de

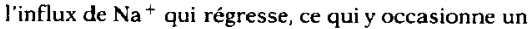
changement du potentiel électrochimique membranaire entraînant une perte sérum - milieu pour $\mathrm{Na}+$ (McWilliams \& Patts, 1978, Fromm 1980). Il en résulte une baisse prononcée en $\mathrm{Na}^{+}$(hyponatriémie) et, secondairement, en $\mathrm{Cl}$ (hypochlorémie) dans le sérum (Muniz et Leviestad, 1979; SNSF, 1981). Quant à l'influx d'ions $\mathbf{H}^{+}$, il peut être neutralisé par le système tampon du sang selon la réaction suivante: $\mathrm{H}^{+}+\mathrm{HCO}_{3} \rightarrow \mathrm{H}_{2} \mathrm{CO}_{3} \rightarrow \mathrm{H}_{2} \mathrm{O}+$ $\mathrm{CO}_{2}$; toutefois, en deça de pH 5,5 dans le milieu, cette neutralisation devient insuffisante et une acidémie sanguine ( $\mathrm{pH} \leqslant 6,9$ ) s'observe (Fromm, 1980 ; Hille, 1982). Suite à la libération accrue de $\mathrm{CO}_{2}$ par le système tampon, la teneur de ce gaz tend à s'élever (hypercapnie) dans le sang: pour atténuer ce phénomène, le poisson accroît sa ventilation respiratoire branchiale en accélérant son rythme de battement operculaire (Janssen \& Randall 1975, Neville $1979 \mathrm{a}$ et b). Cette réaction lui permet aussi d'essayer de compenser la diminution d'entrée d' $\mathrm{O}_{2}$ causée par l'altération des branchies mais, $s^{\prime} i l$ y a acidémie sanguine, cette compensation a peu d'effet car la capacité de l'hémoglobine de transporter l'oxygène est réduite en conditions légèrement acides ; bref, malgré l'effort de ventilation, le poisson risque d'avoir une pénurie d'oxygène (hypoxie) dans le sang (Fromm 1980). Parallèlement à ces changements gazeux dans le système circulatoire, l'hyponatriémie et l'hypochlorémie prédécrites modifient les échanges osmotiques entre le sérum et les cellules : afin d'éviter un gonflement par appel d'eau, celles-ci libèrent des ions $\mathrm{K}^{+}$et des acides aminés, leque] appauvrissement cellulaire a notamment été constaté dans le myocarde qui s'amincit (Daye \& Garside 1980, SNSF 1980). De plus, pour tenter de pallier l'hyponatriémie et l'hypochlorémie, le poisson accroît sa secrétion d'hormones urophysaires (Chevalier 1982), abaisse celle des stéroïdes (Mudge \& 
al 1977) et augmente sa consommation d'oxygène au niveau des branchies en vue d'y amplifier les transports actifs ioniques (Rosseland 1980). Enfin, le dérèglement de l'osmorégulation et le déficit énergétjque métabolique résultant des efforts faits en vue de compenser les effets causés par l'acidité occasionnent plusieurs autres modifications: excrétion réduite des ions et des déchets métaboliques dans les reins, empoisonnement progressif conséquent, activité cardiaque et coordination nerveuse affaiblies, etc... (Daye \& Garside 1980).

Lorsque l'alevin de Salmo salar évolue en tacon et ensuite en saumoneau, tous ces différents phénomènes interreliés se poursuivent et s'amplifient en milieu acide (Daye 1981).

Finalement, à la fin de leur croissance en eau douce, la «smoltification» représente le dernier processus physiologique important pour les jeunes saumons après quelques années peu avant leur descente vers la mer. Au cours de cette étape, les saumoneaux sont très fragiles et peu résistants. Il ne semble pas qu'ils soient affectés par des $\mathrm{pH}$ légèrement acides $(\mathrm{pH} 5,5-6,0)$; ils le deviendraient toutefois à des $\mathrm{pH}$ inférieurs (Saunders \& al. 1980).

L'ensemble des données écotoxicologiques décrites pour le $\mathrm{pH}$ permet d'indiquer qu'un $\mathrm{pH}$ 4,5 - 5,0 peut être considéré comme un seuil pour la survie de Salmo salar mais qu'à long terme, un $\mathrm{pH}$ entre 5,0 et 6,0 est peu favorable au frai, au développement des cufs, à l'éclosion, à l'adaptation des alevins et à leur croissance ultérieure d'autant plus que les effets sont alors cumulatifs. De plus, il semble que le saumon ne puisse développer une résistance aux $\mathrm{pH}$ acides à ces différents stades, comme l'ont montré certains tests d'acclimatation essayés à cette fin (Daye 1980).

Par rapport à ces données, rappelons que les 4 rivières étudiées reçoivent des pluies et des neiges acides ( $\mathrm{pH}$ moyens respectifs : 4,1 et 4,6 ) et qu'à leur tête de bassins, leur très faible alcalinité ne peut complètement neutraliser ces apports acides de telle sorte que leur $\mathrm{pH}$ devient quelque peu inférieur à 6,0 . Cette descente de $\mathrm{pH}$ risque de s'y accentuer et d'y atteindre 5,5 et peut-être moins lors des prochaines années vu que ces rivières sont actuellement en cours d'acidification, comme d'autres à la Côte Nord du fleuve Saint-Laurent (Van Coillie \& al. 1982b), si l'on considère leur teneur en sulfates et leur déficit en alcalinité.
Bref, en intégrant les diverses informations précédentes, il appert que le développement de Salmo salar dans les 4 rivières est actuellement peu menacé par le pH malgré les précipitations acides locales mais que cette menace s'accentuera durant les prochaines années.

\section{4. - Facteur aluminium}

Suite aux èchanges entre les excès d'ions $\mathrm{H}^{+}$des neiges et pluies acides et les alumino-silicates du bouclier canadien, la teneur d'aluminium augmente considérablement au printemps dans les 4 rivières. En effet, elle y atteint alors, en moyenne pondérée par le débit pour les 5 stations d'échantillonnage, $174 \mu \mathrm{g} / \mathrm{L}$ (valeurs brutes entre 39 et $894 \mu \mathrm{g} / \mathrm{L}$ ) par rapport à $61 \mu \mathrm{g} / \mathrm{L}$ en hiver (valeurs brutes entre $16 \mathrm{et}$ $121 \mu \mathrm{g} / \mathrm{L}$ ) et reste ensuite élevée lors de l'été au cours duquel elle équivaut à $158 \mu \mathrm{g} / \mathrm{L}$ (valeurs brutes entre 64 et $236 \mu \mathrm{g} / \mathrm{L}$ ).

En conditions normales, bien qu'il soit difficile de fixer une norme pour des milieux aquatiques variés, on admet généralement que la concentration en aluminium se situe en deçà de $100 \mu \mathrm{g} / \mathrm{L}$ dans les eaux douces (Schofield \& Trojnar 1980, McNelly \& al, 1980).

Comme plusieurs autres régions affectées par les précipitations acides, les 4 rivières concernées renferment dès lors des concentrations anormalement hautes en aluminium. Une situation analogue prévaut au sud de la Norvège où les plans d'eau ont plus de $200{ }_{\mu} \mathrm{g} / \mathrm{L}$ surtout au printemps (SNSF 1980) et au nord-est des Etats-Unis où il y a jusqu'à 320 à 1000 $\mu \mathrm{g} / \mathrm{L}$ dans des milieux aquatiques de la chaine des Adirondack lors de la fonte des neiges (Cronan \& Schofield 1979). Cela se constate aussi en Ontario, plus précisément dans le massif montagneux La Clóche où l'eau renferme 40 à $750 \mu \mathrm{g} / \mathrm{L}$ (Harvey \& al. 1981), ainsi que dans les lacs du parc des Laurentides où il y a 70 à $230 \mu \mathrm{g} \mathrm{Al} / \mathrm{L}$ en moyenne (Potvin 1982).

La teneur en aluminium augmente avec l'acidité dans les eaux de surface (Harvey \& al. 1981, Kramer 1981). Ceci s'explique par la solubilité de l'aluminium qui s'y accroît lorsque le $\mathrm{pH}$ descend sous $\mathrm{pH}$ 6,5. Il est alors dissous sous forme $\mathrm{d}^{\prime} \mathrm{Al}^{+++}$, Al $(\mathrm{OH})^{++}$et $\mathrm{Al}(\mathrm{OH})_{2}{ }^{+}$principalement et d'Al $(\mathrm{OH})_{3}$ et $\mathrm{Al}(\mathrm{OH})_{4}-$ secondairement; à $\mathrm{pH} 5,0-5,2$, les trois premières formes de speciation de l'aluminium 
se retrouvent en parts sensiblement égales nettement supérieures à la quatrième tandis que la der. nière devient inexistante (SNSF 1980). Par ailleurs, au-delà de $\mathrm{pH} 6,5$ où elle s'avère minimale, la solubilité de l'aluminium augmente également mais présente un autre ordre de spéciation, à savoir par ordre decroissant : Al $(\mathrm{OH})_{4}{ }^{-}, \mathrm{Al}(\mathrm{OH})_{3}, \mathrm{Al}(\mathrm{OH})_{2}{ }^{+}$ et $\mathrm{Al}(\mathrm{OH})^{++}$. Ajoutons que l'aluminium se retrouve aussi adsorbé sur des particules en suspension et complexé à de la matière organique dans les eaux naturelles à divers $\mathrm{pH}$; il semble même que ce soit souvent l'aluminium organique qui prédomine dans les eaux naturelles parmi les différentes formes de spéciation du métal (Baker \& Schofield 1980).

La spéciation de l'aluminium présent dans les quatre rivières étudiées n'a pu être précisée dans notre étude préliminaire faite en 1981 car on ne disposait pás à ce moment-là d'ure méthodologie adéquate (Campbell \& al. 1982). Néanmoins, compte tenu de la filtration des échantillons $(\leqslant 0,45 \mu \mathrm{m})$ et des informations précédentes, on peut penser qu'en fonction de leur $\mathrm{pH}$ moyen 6,7 dans leurs portions inférieures et moyennes, le métal se trouve dans celles-ci principalement sous les formes suivantes: organique, $\mathrm{Al}(\mathrm{OH})_{3}$ et $\mathrm{Al}(\mathrm{OH})_{4}{ }^{-}$. Si, tel que présumé plus haut, leur $\mathrm{pH}$ devient plus acide ( $\mathrm{pH} \leqslant$ $6,0)$ dans leurs portions supérieures, l'aluminium serait alors réparti dans celles-ci de façon différente : organique, $\mathrm{Al}(\mathrm{OH})_{2}{ }^{+}, \mathrm{Al}(\mathrm{OH})^{++}, \mathrm{Al}^{+3}$ et à moindre échelle, $\mathrm{Al}(\mathrm{OH})_{3}, \mathrm{Al}(\mathrm{OH})_{4}{ }^{-}$.

La toxicité de toutes ces formes d'aluminium demeure encore mal connue. Il a tou tefois été montré que l'aluminium s'avère au moins deux fois plus toxique sous forme organique pour Salvelinus fontinalis (Driscoll \& al. 1980). L'influence de la spéciation de l'aluminium inorganique sur la toxicité de ce metal a également été décelée indirectement par des bio essais à différents $\mathbf{p H}_{2}$ tels que ceux réalisés par Schofield \& Trojnar (1980) avec des alevins de Salvelinus fontinalis. A PH 4,0, le TL 50 (temps létal pour $50 \%$ des individus) ne change pas, à savoir 5 jours, pour des teneurs variant de 0 à 1000 $\mu \mathrm{g} / \mathrm{L}$, ce qui indique que l'action toxique est davantage due à l'acidité qu'à l'aluminium à ce $\mathrm{pH}$ auquel le métal ajouté se trouve à $75 \%$ sous forme $\mathrm{d}^{\prime} \mathrm{Al}^{+++}$; il y aurait alors un antagonisme compétiteur d'action des ions $\mathrm{H}^{+}$vis à vis des ions $\mathrm{Al}^{+++}$. A pH 4,4, par contre, la toxicité de l'aluminium apparait : Le TL 50 équivaut respectivement à 10 et 2 jours pour des teneurs de 0 et $500 \mu \mathrm{g} / \mathrm{L}$. A
pH 4,9 $-5,2$, l'effet des ions $\mathrm{H}^{+}$régresse et celui de l'aluminium prédomine : 90 à $100 \%$ des alevins survivent plus de 100 jours dans 0 et $100 \mu \mathrm{g} / \mathrm{L}, 63 \%$ à $90 \%$ des alevins résistent 14 à 23 jours dans 250 $\mu \mathrm{g} / \mathrm{L}$ et $50 \%$ d'entre eux meurent en 2 jours dans $500 \mu \mathrm{g} / \mathrm{L}$. L'accroissement de la toxicité de l'aluminium avec la hausse du $\mathrm{pH}$ entre 4,0 et 5,2 suggère que l'antagonisme diminue entre les ions $\mathbf{H}^{+}$, et $\mathrm{Al}^{+++}$; de plus, ce sont surtout les ions $\mathrm{Al}(\mathrm{OH})^{++}$ et $\mathrm{Al}(\mathrm{OH})_{2}{ }^{+}$qui peuvent alors occasionner une toxicité. Au-delà de pH 5,7 jusqu'à 6,5 les trois dernières formes de l'aluminium deviennent moins abondantes que $\mathrm{Al}(\mathrm{OH})_{3}$ et $\mathrm{Al}(\mathrm{OH})_{4}^{-}$et la solubilité du métal diminue; sa toxicité est alors réduite.

De tels bioessais indiquent que la toxicité de l'aluminium inorganique se manifeste surtout à pH 5,2 $-5,7$ pour Salvelinus fontinalis (Baker \& Schofield 1980; Schofield et Trojnar 1980) de même que pour Salmo trutta (Muniz \& Leivestad 1980). A pH 5,3 5,6, sa CL50-7 jours (concentration létale pour $50 \%$ des individus durant 7 jours de bioessais) équivaut à $480 \mu \mathrm{g} \mathrm{Al} / \mathrm{L}$ pour Salvelinus fontinalis dans une eau synthétique ayant une assez faible alcalinité ( $\leqslant 800$ $\mu$ éq/L) et s'élève à $5300 \mu \mathrm{g} \mathrm{Al} / \mathrm{L}$ lorsqu'on ajoute 10 $\mathrm{mg} / \mathrm{L}$ de matière humique, laquelle complexe facilement l'aluminium et le rend ainsi moins toxique (Thellen \& Van Coillie 1982). Au niveau sous-létal, cette espèce a une réaction de fuite significative ( $\geqslant 20 \%$ des indidivus) à partir de $140 \mu \mathrm{g} \mathrm{Al} / \mathrm{L}$ à $\mathrm{pH}$ 5,5 pendant des tests d'évitement (Thellen \& Van Coillie 1982). En fonction des diverses informations précédentes, on peut donc considérer que l'aluminium inorganique devient toxique à $150 \mu \mathrm{g} / \mathrm{L}$ à $\mathrm{pH}$ 5,5 vis-à-vis de Salvelinus fontinalis.

Pour Salmo salar, il y peu de données relatives à la toxicité de l'aluminium. A pH 5,3-5,5 avec alcalinité $\leqslant 80{ }_{\mu} \mathrm{e} q / \mathrm{L}$, la LC $50-7$ jours de ce métal sous forme inorganique pour les alevins de cette espèce correspond à $170 \mu \mathrm{g} \mathrm{Al} / \mathrm{L}$ et, à partir de $90 \mu \mathrm{g} \mathrm{Al} / \mathrm{L}$, un stress respiratoire sous-létal se manifeste de façon évidente (Van Coillie \& Thellen 1982). 11 sem. ble donc que le saumon soit beaucoup plus sensible à la toxicité de l'aluminium inorganique que $\mathrm{Sal}$. velinus fontinalis : à $\mathrm{pH} 5,5$, une concentration voisine de $100 \mu \mathrm{g} \mathrm{Al} / \mathrm{L}$ lui serait néfaste.

L'aluminium provoque une série d'altérations dans les branchies des poissons à pH 5,0-5,5:prolifération cellulaire aux extrémités des filaments, desquamation et nécrose des cellules épithéliales d'échange, œedème, replis des lamelles, gonflement 
des cellules à mucus et accumulation périphérique de celui-ci (Grahn 1980, Schofield \& Trojnar 1980, Playle 1982, Thellen \& Van Coillie 1982). Bien que ces altérations soient partiellement dues au $\mathrm{pH}$ modérément acide (voir plus haut), elles sont aussi causées et/ou amplifiées par l'aluminium vu que celui-ci s'y bioaccumule selon un facteur multiplicateur variant de 5 à 33 fois avec sa teneur, la durée et le pH (Grahn 1980, Van Coillie \& Thellen 1982). Les modifications induites occasionnent une réduction des influx de $\mathrm{Na}^{+}$et $\mathrm{CL}^{-}$avec une hyponatriémie et une hypochlorémie subséquentes : pour tenter d'y remédier, il y a hyperventilation branchiale et amplification de la consommation d'oxygène (Muniz \& Leivestad 1980, Schofield \& Trojnar 1980, Thellen \& Van Coillie 1982). De plus, bien que l'aluminium pénètre peu dans le poisson, il s'y retrouve entre autres dans ses muscles et y déclanche des réactions telles qu'une synthèse accrue de protéines, lesquelles pourraient correspondre à des anticorps et/ou à des hormones (Van Coillie \& Thellen, 1982).

$\mathrm{Si}$ l'on considère que cette toxicité de l'aluminium se manifeste à partir de $100 \mu \mathrm{g} \mathrm{Al}$ inorganique $/ \mathrm{L}$. chez Salmo salar (voir plus haut), la survie de cette espèce dans les 4 rivières étudiées peut sembler menacée vu qu'elles ont des teneurs moyennes d'aluminium s'échelonnant de 158 à $174 \mu \mathrm{g} \mathrm{Al} / \mathrm{L}$ et qu'on a constaté des maxima à 236 ef $894 \mu \mathrm{g} \mathrm{Al} / \mathrm{L}$ à $\mathbf{E S}_{2}$ (Brouard \& al., 1982) respectivement en été et au printemps. Toutefois, l'indication de $100 \mu \mathrm{g} \mathrm{Al} / \mathrm{L}$ vaut pour un $\mathrm{pH}$ voisin de 5,5 . Or le $\mathrm{pH}$ moyen fluctue entre 6,4 et 7,0 dans les 4 rivières. A ce niveau de $\mathrm{pH}$, l'aluminium devient toxique à des concentrations vraisemblablement assez supérieures à $100 \mu \mathrm{g}$ Al inorganique /L car ses formes de spéciation son $t$ différentes et moins néfastes (voir plus haut) dans un milieu quasiment pas acide. Certes, le $\mathrm{pH}$ peut être quelque peu plus acide ( $\mathrm{pH} \leqslant 6,0$ ) dans les portions supérietures des 4 bassins au printemps tel que mentionné précédemment mais l'apport d'aluminium s'avère plus faible dans celles-ci qu'en aval à cette saison (124 $\mu \mathrm{g} \mathrm{Al} / \mathrm{L}_{\text {à ES}}$ et $355 \mu \mathrm{g} \mathrm{Al} / \mathrm{L}_{\text {à ES}}$ ) et, de façon plus générale, durant l'ensemble de l'échantillonnage (114 $\mu \mathrm{g} \mathrm{Al} / \mathrm{L}$ à ES $\mathrm{ES}_{1}$ et $197 \mu \mathrm{g} \mathrm{Al} / \mathrm{L}$ à $\mathrm{ES}_{2}$ ). De plus, les eaux dẹs 4 rivières contiennent des teneurs relativement élevées de carbone organique après filtration $(3,8$ à $8,5 \mathrm{mg} / \mathrm{L})$ par rapport à leur oligotrophie (Brouard $\&$ al. 1982) et les concentrations déterminées pour l'aluminium soluble renferment dès lors sans doute une part organique importante, laquelle est beaucoup moins toxique que celle de nature inorganique. En tenant compte de ces divers facteurs, il faut at ténuer le risque écotoxicologique lié à l'aluminium dans les 4 rivières pour Salmo salar. Cependant, dans les conditions actuelles, ce risque y apparaît assez prononcé à l'aval des bassins. De plus, nul doute que l'amplification graduelle des précipitations acides dans la région entraînera celle de la toxicité de l'aluminium dans ses eaux de surface.

\section{5. - Autres métaux traces}

Le ruissellement des eaux des précipitations acides à travers les sols peut occasionner non seulement une libération accrue d'aluminium mais aussi un relachement de quantités anormalement élevées en fer, manganèse, cuivre, zinc, mercure, cadmium et plomb dans les eaux de surface (Harvey \& al 1981). Cela a été constaté entre autres dans des eaux affectées par des précipitations acides au sud de la Scandinavie (Almer \& al. 1978, Dickson 1980) et en Ontario près de Sudbury et dans le massif montagneux La Cloche (Harvey \& al. 1981, Harvey \& Fraser 1982).

Une augmentation analogue de certains métaux survient aussi au printemps dans les 4 rivières étudiées. En effet, par rapport à celles de l'hiver, les teneurs en fer, manganèse et cuivre s'y sont élevées respectivement de 111 à $206 \mu \mathrm{g} \mathrm{Fe} / \mathrm{L}, 4,7$ à $10,4 \mu \mathrm{g}$ $\mathrm{Mn} / \mathrm{L}$ et 1,0 à $6,8_{\mu} \mathrm{g} \mathrm{Cu} / \mathrm{L}$ en moyenne au printemps et ont alors atteint à $\mathrm{ES}_{2}$ des maxima de $376 \mu \mathrm{g}$ $\mathrm{Fe} / \mathrm{L}, 31,0 \mu \mathrm{g} \mathrm{Mn} / \mathrm{L}$ et $27,5 \mu \mathrm{g} \mathrm{Cu} / \mathrm{L}$ (Brouard \& al. 1982). Pour la majorité des échantillons pris à cette saison, elles dépassaient celles qu'on retrouve habituellement dans des eaux oligotrophes, soit $200 \mu \mathrm{g}$ $\mathrm{Fe} / \mathrm{L}, 3 \mu \mathrm{g} \mathrm{Mn} / \mathrm{L}$ et $5 \mu \mathrm{g} \mathrm{Cu} / \mathrm{L}$ (Alabaster \& Lloyd 1980, Mc Nelly \& al. 1980, Harvey \& al. 1981). Ceci n'a pas été constaté pour le zinc, le mercure, le cadmium, le nickel et le plomb, lesquels avaient des concentrations inférieures à celles qui induisent des toxicités sous-létales chez les salmonidés, à savoir 100 $\mu \mathrm{g} \mathrm{Zn} / \mathrm{L}, 2 \mu \mathrm{g} \mathrm{Hg} / \mathrm{L}, 2 \mu \mathrm{g} \mathrm{Cd} / \mathrm{L}, 100 \mu \mathrm{g} \mathrm{Ni} / \mathrm{L}$ et $50 \mu \mathrm{g}$ Pb/L (EPA, 1973; Van Coillie 1977; Alabaster \& Lloyd 1980). Dans ces conditions, on ne considèrera que le fer, le manganèse et le cuivre présents dans ces rivières pour le suite de l'exposé.

La spéciation de ces trois métaux dans le milieu aquatique s'avère déterminante pour leur toxicité, comme c'est le cas pour l'aluminium; à ce titre, elle mérite d'être précisée à partir des données connues 
à ce sujet. En ce qui concerne le fer, il se retrouve généralement sous les trois formes suivantes par ordre décroissant dans les eaux naturelles : précipité en particules (grandeur $\geqslant 0,01 \mu \mathrm{m}$ ), associé avec de la mat tère organique et dissous comme composé inorganique (Harvey \& al. 1981). C'est surtout cette dernière forme qui est toxique ; elle contient principalement des hydroxides (Alabaster \& Lloyd 1980). La solubilité du fer est minimale à $\mathrm{pH}$ 7,0 et s'accroît de $\mathrm{pH} 7,0$ à 4,0 davantage sous forme $\mathbf{F e}$ $(\mathrm{OH})_{2}{ }^{+}$que $\mathrm{Fe}(\mathrm{OH})^{++}$et surtout $\mathrm{Fe}^{+++}$quil ne devient évident que sous pH 5,5 (Harvey \& al. 1981). Ceci explique que son seuil de toxicité létale diminue avec le $\mathrm{pH}$ pour Salvelinus fontinalis : de fait, il se chiff re respectivement à 1,75 et $0,39 \mathrm{mg} / \mathrm{L}$ à $\mathrm{pH}$ 6,0 et 5,5 pour cette espèce (Decker \& Menendez 1974). Similairement au fer, le manganèse existe sous forme précipitée, organique et minérale et sa toxicité est surtout reliée à sa forme minérale dis. soute, laquelle est amplifiée à des $\mathrm{pH}$ inférieurs a 6,0 (Almer \& al. 1978); son seuil de toxicité létale pour les salmonidés se situe au-delà de $150 \mu \mathrm{g} / \mathrm{L}$ (EPA 1973). Enfin, pour le cuivre, il y a trois formes majeu. res de spéciation, lesquelles se présentent dans les eaux naturelles selon l'ordre décroissant suivant : particulaire comme $\mathrm{CuCO}_{3}$, complexé à des composés chimiques ou dissous en ions $\mathrm{Cu}^{++}, \mathrm{Cu}(\mathrm{OH})^{+}$ et $\mathrm{Cu}(\mathrm{OH})_{2}$. Un $\mathrm{pH}$ acide tend à augmenter $\mathrm{Cu}^{+}+$ au détriment de $\mathrm{Cu}(\mathrm{OH})^{+}, \mathrm{Cu}(\mathrm{OH})_{2}$ et du $\mathrm{Cu}\left(\mathrm{CO}_{3}\right)$ (Alabaster \& Lloyd 1980). La toxicité du cuivre serait due aux ions $\mathrm{Cu}^{++}$et également aux particules $\mathrm{Cu}$ $\mathrm{CO}_{3}$ selon Shaw \& Brown (1974) et exerce un effet létal à partir de $25 \mu \mathrm{g} / \mathrm{L}$ chez les salmonidés (Alabaster \& Lloyd 1980).

Les trois métaux considérés agissent surtout au niveau des branchies des poissons. Le fer et le manganèse ont tendance à s'y précipiter en surface (Alabaster \& Lloyd, 1980) tandis que le cuivre peut s'y bioaccumuler et induire des détériorations des cellules épithéliales en même temps qu'une réduction des cellules à mucus (Pequignot \& al. 1975, Alabaster \& Lloyd 1980) : de façon générale, un dérèglement de l'osmorégulation et des échanges respira. toires en résulte. Le manganèse remplace aussi partiellement le calcium dans les structures ostéoïdes, ce qui les affaiblit (Harvey \& Fraser 1982). En outre, le cuivre réduit l'activité de certaines enzymes telles que la glutamo-oxalo-acétate transaminase du plasma chez. Salvelinus fontinalis (McKim \& Benoît 1971). En se liant avec l'ADN (acide désoxyribonu- clique), ce métal peut aussi provoquer indirectement une inhibition de synthèse de macromolécules nécessaires à un développement ultérieur chez les embryons de Salmo salar et Salvelinus fontinalis (Van Coillie \& al. 1975). Ces diffërens effets toxiques apparaissent au niveau sous-létal à partir de $5 \mu \mathrm{g}$ $\mathrm{Cu} / \mathrm{L}, 100 \mu \mathrm{g} \mathrm{Mn} / \mathrm{L}$ et $300 \mu \mathrm{g} \mathrm{Fe} / \mathrm{L}$ à pH 5,5-7,0 (Van Coillie al 1975, Alabaster \& Lloyd 1980, McNelly \& al. 1980, Harvey \& Fraser, 1982).

Ces concentrations ne se retrouvent que partiellement dans les 4 rivières étudiées. En effet, celles de Fe dépassent quelque peu $300 \mu \mathrm{g} \mathrm{Fe} / \mathrm{L}$ seulement à $E_{2}$ au printemps, celles de Mn s'avèrent très inférieures à $100 \mu \mathrm{g} \mathrm{Mn} / \mathrm{L}$ et celles de $\mathrm{Cu}$ sont souvent plus élevées que $5 \mu \mathrm{g} / \mathrm{L}$ au printemps et, à moindre degré, en été sans toutcfois atteindre le seuil létal de $25 \mu \mathrm{g} / \mathrm{L}$ sauf en une occasion à $\mathrm{ES}_{2}(27,5$ $\mu \mathrm{g} / \mathrm{L}, 10 \mathrm{mai}$ 1981). Bref, excepté pour le cuivre, les teneurs actuelles en métaux traces autres que l'aluminium dans les 4 rivières ne représentent pas un danger écotoxicologique pour les salmonidés locaux.

\section{6. - Conclusion}

Il y a 3 possibilités écotoxicologiques actuelles relićes aux précipitations acides régionales pour Salmo salar dans les 4 rivières étudièes.

- La faible alcalinité des eaux oligotrophes diminue d'aval en amont dans les bassins et devient insuffisante à leurs têtes pour neutraliser complètement les apports acides des pluies et neiges régionales ( $\mathrm{pH}$ moyens respectifs : 4,1 et $4,6)$ : leur $\mathrm{pH}$ y descend quelque peu sous 6,0 à la fonte des neiges. Cette tendance risque de s'accentuer avec la poursuite des précipitations acides lors des prochaines années car ces rivières sont en cours d'acidification si l'on considère leur teneur en sulfates et leur déficit en alcalinité : leur pH pourrait alors s'approcher de 5,5, lequel s'avère néfaste au développement des ceufs et alevins de saumon.

- La teneur en aluminium est anormalement élevée au printemps et en été dans les 4 rivières vraisemblablement à cause d'un lessivage des alumino-silicates du bouclier canadien par les précipitations acides. Elle dépasse $100 \mu \mathrm{g} \mathrm{Al} / \mathrm{L}$, valeur à partir de laquelle le saumon est sensible à la toxicité du métal à pH 5,5. Toutefois, si l'on tient compte de leur $\mathrm{pH}$ moyen actuel $(6,4$ 
à 7,0 ) et de leur matière organique à laquelle se complexe $\mathrm{Al}$, le risque écotoxicologique que représente l'aluminium de ces rivières pour Salmo salar doit être atténué. Sa concentration y augmentant d'amont vers aval suite à un apport progressif des bassins versant, ce risque écotoxicologique est surtout présent dans les portions inférieures des rivières $(355 \mu \mathrm{g} \mathrm{Al} / \mathrm{L}$ en moyenne à l'ernbouchure de la rivière des Escoumins au printemps).

- Les 4 rivières ont également une nette augmentation des concentrations en fer, manganèse et cuivre au printemps : dans la majorité des cas, celles-ci y dépassent alors les teneurs qu'on retrouve dans les eaux oligotrophes non affectées par les précipitations acides, soit $200 \mu \mathrm{g}$ $\mathrm{Fe} / \mathrm{L}, 3 \mu \mathrm{g} \mathrm{Mn} / \mathrm{L}$ et $5 \mu \mathrm{g} \mathrm{Cu} / \mathrm{L}$. le risque écotoxicologique de ces dépassements pour le saumon est cependant limité actuellement au cuivre seulement dans ces rivières.

Ces 3 possibilités écotoxicologiques sont surtout présentes à la fonte des neiges pendant que le saumon local est au début du stade alevin, particulièrement sensible au cours du développement de l'espèce; elles représentent donc une réelle menace pour le maintien futur de celle-ci dans les 4 rivières.

\section{Travaux cltés}

Alabaster (J.S.) \& Lloyd (R.) 1980. - Water qualiry criteria for freshwater fish. Food and Agriculture Organization (United States). Butterworths Co. Publishers, London, 297 p.

Almer (B.), Dickson (W.), Ekstrom (C.) \& Hornstrom (E.), 1978. Sulfur pollution and the aquatic ecosystem. Dans: Sulfur in the environment. Part II : Ecological impacts. J.O. Nriagu (ed.) J. Wiley and Sons Inc., New York : 271-311.

Altshuller (A.) \& McBean (G.). 1980. - Le transport à grande distance de polluants atmosphériques. Deuxième rapport du groupe consultatif de recherche Etats-Unis - Canada. Environnement Canada, 46 p.

APHA-AWWA-WPCF (American Public Health Association, American Water Works Association, Water Pollution Control Federation) 1980.-Standord methods for the examination of water and waste water. 15th ed. American Public Health Association Washington, 1134 p.

Baker (J.P.) \& Schofield (C.L.). 1980-Aluminium toxicity fo fish as related to acid precipitation and Adirondack surface water quality. Dans : Ecological impact of acid precipitation. SNSF. project. Sandefjord, Norway: 292.293.

Bat tle (H.1.). 1944. - The embryology of the atlantic salmon Saimo salar Can. J Res. 22 D. 105-124

Brouard (D.) \& Lachance (M.). 1982.- Qualite physico-chimique printanière des eaux de surface et des precipitations au secteur amont de la rivière des Escoumins (1982). Rapport d'etape pour Pêches et Océans Canada, Région du Ouébec, 26 p.
Brouard (D.) Lachance (M.). Shooner (G.) \& Van Coillie (R.). 1982.Sensibilité a l'acidification de quatre rivières a saumons a la Côte Nord du fleuve Saint-Laurent (Ouébec). Rapport technique canadien des Sciences Halieutiques et Aquatiques, no $1109 \mathrm{~F}, 56 \mathrm{p}$.

Brouard (D.), Lachance (M.) \& Van Coillie (R.). 1981 - Caractérisation physico-chimique hivemale des eaux de quatre rivières à saumons de la Côte-Nord du fleuve Saint-Laurent. Petit Saguenay, Ste-Marguerite, des Escoumins et des Petits Escou. mins. Rapport pour Ministère des Péches et Océans du Canada, $61 \mathrm{p}$.

Bua (B.) \& Snekik (E.). 1972. - Hatching experiments with roe from salmonid fishes, 1966-1971: effects of acidity and dissolved salts. Vann, $7: 86-93$

Campbell (P.G.), Bisson (M.), Boisvert (I.), Bougie (R.), Tessier (A.) \& Villeneuve (J.P.). 1982.- Méthodologie analytique pour déterminer la spéciation de l'aluminium dans des eaux lacustres en voie d'acidification. Institut National de la Recherche Scientifique, INRS-Eau, Rapport 145: 113 p.

Carrick (T.R.). 1979. - The effects of acid water on the hatching of salmonid eggs. J. Fish Biol, 14: 165.172.

Carter (W.M.)-Le saumon de l'Atlantique au Ouébec : rapport sur le saumon et plan directeur pour l'aménagement de cette ressource. Rapport pour ministère du Tourisme de la Chasse et de la Pèche du Québec, $236 \mathrm{p}$.

Chevalier (G.). 1982. - Effets des eaux acides sur l'activité de l'urophyse chez Salvelinus fontinalis (en rédaction).

Cronan (C.S.) \& Schof ield (C.L.). 1979, - Aluminium leaching response to acid precipitation : effects on highelevation watersheds in the northeast. Science, $204:$ 304-306.

Daye (P.G.). 1980.-- Attempts to acclimate embryos and alevins of Atlantic salmon, Salmo salar, and rainbow trout. $S$ gairdmeri. to low ph. Can. J. fish. Aquat. Sci., 37: 1035-1038.

Daye (P.G.). 1981.- The impact of acid precipitation on the physiology and toxicology of fish. Proc. Intern. Conf. on Acid Rain and the Atlantic Salmon, International Atlantic Salmon Foundation. Special publication $10: 29-34$.

Daye (P.G.) \& Carside (E.T.). 1980.- Structural alterations of embryos and alevins of Atlantic salmon, Salmo salar L., induced by continuous or short-term exposures to acidic levels of pH. Can. J. Zool., 58 ; $27-43$.

Decker (C.) \& Menendez (R.). 1974.- Acute toxicity of iron aluminum to brook trout. Proc. W. Va Acad. Sci., 46: 159-167.

Dickson (W.). 1980.- Properties of acidified water. Dans : Ecological impact of acid precipitation. SNSF-project. Sandefjord, Norway: $75-83$.

Dillon (P.J.), Jeffries (D.S.), Scheider (W.A.) \& Yan (N.D.). 1980.Some aspects of acidification in southern Ontario. Dans : Ecological impact of acid precipitation. SNSF.project. Sandefjord, Norway, 212-213.

Driscoll (C.T.), Baker (J.P.), Bisogni (J.) \& Schofield (C.L.). 1980.Aluminium speciation in dilute acidified waters and its effects on fish. Nature (London), $284: 161-164$.

EPA (Environmental Protection Agency). 1973, - Water quality criteria data book. Volume 5 : Effects of chemicals on aquatic life. Water Pollution Control Research Series. U.S. Govermment Printing Office, Washington, D.C. No 18050 HLA $09 / 73$.

Farmer (G.J.), Goff (T.R.). Ashfield (D.) \& Samant (H.S.). 1980.Some effects of the acidification of Atlantic salmon rivers in Nova Scotia. Can. Tech. Rep. Fish. Aquat. Sci. 972.13 p.

Fromm (P.0.). 1980.- A review of some physiological and toxicological responses of freshwater fish to acid stress. Env. Biol. Fish. $5: 79-93$.

Grahn (O.). 1930.- Fish kills in two moderately acid lakes due to high aluminum concentration. Dans : Ecological impact of acid precipitation SNSF-project. Sandefjord, Norway. 310-311.

Grande (M.), Muniz (I.P.) \& Anderson (S.). 1978.- Relative tolerance of some salmonids to acid waters, Verh. Intemat. Verein. Limnol., 20 : 2076-2084 
Harvey (H.H.) \& Fraser (G.A.). 1982. - Freshwater acidification and the elevation of manganese in water and fishes. Symposium sur l'acidification des eaux du bouclier canadien, 2-4 avril 1982, Tononto.

Harvey (H.H.), Pierce (R.C.), Dillon (P.J.) Kramer (J.R.) \& Whelpdale (D.M.) 1981 . Acidification in the canadian aquatic environment : scientific criteria for assessing the effects of acidic deposition on aquatic ecosystems. National Research Coun. cil of Canada, NRCC no $18475,369 \mathrm{p}$.

Hille (S.). 1982 - A literature review of the blood chemistry of rainbow trout, Salmo gairdneri Richardson. I. Fish. Biol., 20 : 535.569 .

Hovington (L.), Premont (P.), Perron (S.), Villeneuve (M.) \& Gauthier (L.). 1978- Etude des frayères à saumons dans la rivière Escoumins. Rapport ministère Emploi et Immigration du Canada, 39 p.

Jansen (R.G.) \& Randall (D.J.). 1975. - The effects of changes in pH and $P_{\mathrm{CO}}$ in blood and water on breathing in rainbow trout, Salmo gairdneri. Respir. Physiol., $25: 235-245$.

Johansson (N.), Runn (P.) \& Milbrink (G.). 1977. - Early development of three salmonid species in acidifted water. Zoon, 5 : $127 \cdot 132$.

Jones (H.G.) « Bisson (M.). 1980_- Lac Laflamme : qualité de l'eau et de la neige. Institut National de la Recherche scientifique, INRS-Eau, rapport 123.91 p.

Kramer (J.R.), 1981.-Aluminium : chemistry, analysis and biology. Mc Master University. Environmental Geochemistry report 1981-1982, $174 \mathrm{p}$.

Kwain (W.H.). 1975. - Effects of temperature on development and survival of rainbow trout, salmo gairdneri. in acid waters. $J$. Fish. Res. Board Can., $32:$ 493-497.

Lachance (M.), Brouard (D.), Van Coillie (R.) \& Dutil (J.). 1982.Physico-chimie des eaux de la rivière Sainte-Marguerite (Québec) en période de fonte des neiges (soumis pour publication).

Lee (R.M.) \& Gerking (S.D.). 1980.- Sensitivity of fish eggs to acid stress. Water Res., 14:1679-1681.

Leivestad (H.), Hendrey (G.), Muniz (I.P.) \& Snekik (E.). 1976.Effects of acid precipitation on freshwater organisms. Dans : Impact of acid precipitation on forest and freshwater ecosystems in Norway. SNSF-project. Norway. FR6/76: 87-111.

Lockhart (W.L.) \& Lutz (A.). 1977. - Preliminary biochemical observations of the fishes inhabiting an acidified lake in Ontario, Canada. Intem. J. Water Soit Pollution, $7: 317.332$.

McKim (J.M.) \& Benoit (D.A.). 197I. - Effects of long.term exposures to copper on survival, growth and reproduction of brook trout Salvelinus fontinalis. J. Fish. Res. Board Can. $28: 655-662$.

McNelly (R.N.), Neimanis (V.P.) \& Dwyer (L.). 1980. - Guide des parametres de la qualité des eaux. Rapport pour Environnement Canada. Direction générale des eaux intérieures, $79 \mathrm{p}$.

McWilliams (P.G.) \& Potts (W.T.). 1978. - The effects of $\mathbf{p H}$ and calcium concentrations on gill potentials in the brown trout Salmo trutia. J. Comp. Physiol., 126: 277-286.

Menendez (R.). 1976. - Chronic effects of reduced pH on brook trout Salvelinus fontinalis. J. Fish. Res. Board Can., 33: 118-123.

Mudge (J.E.), Dively (J.L.), Neff (W.H. \& Anthony (A.), 1977,- Interrenal histochemistry of acidexposed brook trout Salvelinus tontinalis. Gen. Comp. Endocr., 31 : 208-215.

Muniz (1.P.) \& Leivestad (H.). 1979. - Long term exposure of brook trout to acidic water. SNSF-project. Sandefjord. Norway. RR77/79, 32 p

Muniz (I.P.) \& Leivestad (H.). 1980. - Toxic effects of aluminium on the brown trout, Salmo truta. Dans : Ecological impact of acid precipitation. SNSF-project. Sandefjord, Norway.: 320-321.
Nelson (J.A.) 1982.- Physiological observations on developping trout, Salmo gairdmeri Richardson, exposed to low $\mathrm{pH}$ and varied calcium ion concentrations J. Fish. Biol., $20: 359-379$.

Neville (C.M.). 1979 a.-Ventilatory response of rainbow trout Salmo gairdner to increased $\mathrm{H}^{+}$ion concentration in blood and water. Comp. Biochem. Physiol, 63A : 373-376.

Neville (C.M.). 1979 b. - Sublethal effects of environmental acidification on rainbow trout Salmo gairdneri. J. Fish. Res. Bd. Can. $36: 84-87$.

Pequignot (I.), Labat (R.) \& Chatelet (A.). 1975_- Action du sulfate de cuivre sur les cellules à mucus de l'alevin de truite Salmo irideus. J. Eur. Toxicol, $8: 52-56$.

Peterson (R.H.) Daye (P.G.) \& Metcalfe (J.L.). 1980_- Inhibition of Atlantic salmon Salmo salar hatching at low pH. Can J. Fish. Aquat. Sci., $37: 770-774$

Playle (R.). 1982. - Toxicité de l'aluminium chez les ménés en eaux douces artificielles. Symposium sur l'acidification des eaux du bouclier canadien, 2.4 ayril 1982, Toronto.

Potvin (P.). 1982. - Relations pH-aluminium pour les lacs du parc des Laurentides. Symposium sur l'acidification des eaux du bouclier canadien, 2-4 avril 1982, Toronto.

Robinson (G.D.), Dunson (W.A.), Wright (J.E.) \& Mamolito (G.E.) 1976. - Differences in low $\mathrm{pH}$ tolerance among strains of brook trout Salvelinus fontinalis. J. Fish. Biol, 8 : 5-17.

Rosseland (B.O.), 1980.- Effects of acid water on metabolism and gill ventilation in brown trout Salmo trutta and brook trout Salvelinus fontinalis. Dans : Ecological impact of acid preci. pitation. SNSF-project. Sandefjord, Norway: 348.349 .

Ruby (S.M.), Aczel (J.) \& Craig (G.R.). 1979. - The effects of depres. sed $\mathrm{pH}$ on oogenesis in flagfish lordanella floridae. Water Res. $11: 757-762$

Saunders (R.L.), Henderson (E.B.) \& Hormin (P.R.). 1980- Effects of low pH on smolting Atlantic salmon. Fifth Annual Atlantic Region Fish Health Workshop. Halifax, October 14-16, 1980.

Schofield (C.L.) \& Trojnar (J.R.). 1980.-Aluminum toxicity to fish in acidified waters. In : Polluted rain. Plenum Press, New-york. Vol. $17: 347-366$

Sahw (T.L.) \& Brown (V.M.). 1974.- The toxicity of some forms of copper to rainbow trout. Water research, $8: 377-382$.

SNSF-project 1980. - Acid precipitation : effects on forest and fish. Final report of the SNSF-project, 1972-1980. Overrein, L.N., Seip, H.M., Tollan, A. (eds). Sandefjord, Norway, RR19/80, $175 \mathrm{p}$.

Thellen (C.) \& Van Coillie (R.). 1982.- Sensibiité écotoxicologique des salmonidés à l'aluminium en relation avec des conditions physico-chimiques acides. Rapport pour Pêches et Océans. Canada (soumis pour publication).

Van Coillie (R.). 1977.- Effets subletaux des métaux lourds dans les cufs des poissons d'eaux douces. These de doctorat d'Etat Université de Toulouse, 352. p.

Van Coille (R.). Brouard (D.), Lachance (M.) \& Vigneault ( $($.) 1982a. - Effets physico-chimiques des précipitations acides sur 4 rivières à saumons à la Côte Nord du tleuve SaintLaurent. Eau du Ouébec (sous presse).

Van Coillie (R.), Duval (J.) \& Deschênes (C.). 1982b. - Retombées des précipitations acides au bassin supérieur de la rivière Moisie (soumis pour publication).

Van Coillje (R.), Jones (G.), Poirier (D.), Goupil (M.). 1975. - Effets sous-létaux du cuiv re et du zinc chez les oufs de salmonidés. In : Proc. Intern. Conf. Heavy Metals in the Environment. Nat Res. Council Canada. Volume C: $21 \cdot 24$

Van Coillie (R.) \& Thellen (C.). 1982.-Bioessais de toxicité de l'aluminium avec le saumon de l'Allantique Salmo salar, l'omble de fontaine Salvelinus fontinalis et la truite arc-en-ciel Salmo gairdneri. Symposium sur l'acidification des eaux du bouclier canadien, 24 avril 1982, Toronto. 